\title{
Minimally doubled fermions and spontaneous chiral symmetry breaking
}

\author{
Rudina Osmanaj (Zeqirllari), ${ }^{1, \star}$ Dafina Hyka (Xhako) ${ }^{2}$ \\ ${ }^{1}$ University of Tirana, Faculty of Natural Sciences, Department of Physics \\ ${ }^{2}$ Polytechnic University of Tirana, FIMF, Department of Physics
}

\begin{abstract}
Chiral symmetry breaking in massless QCD is a very important feature in the current understanding of low energy physics. Low - lying Dirac modes are suitable to help us understand the spontaneous chiral symmetry breaking, since the formation of a non zero chiral condensate is an effect of their accumulation near zero. The Banks Casher relation links the spectral density of the Dirac operator to the condensate with an identity that can be read in both directions. In this work we propose a spectral method to achieve a reliable determination of the density of eigenvalues of Dirac operator near zero using the Gauss - Lanczos quadrature. In order to understand better the dynamical chiral symmetry breaking and use the method we propose, we have chosen to work with minimally doubled fermions. These kind of fermions have been proposed as a strictly local discretization of the QCD fermions action, which preserves chiral symmetry at finite cut-off. Being chiral fermions, is easier to work with them and their low - lying Dirac modes and to understand the dynamical spontaneous chiral symmetry breaking.
\end{abstract}

\section{Introduction}

Symmetries and their breakings are a very important part of the modern physics. Studying symmetry breaking is as interesting as studying symmetry itself. Spontaneous chiral symmetry breaking is, apart from colour confinement, the most important physical aspect of strong interactions. How does the chiral symmetry breaking happen microscopically? For many years, physcists have relied on models to understand this. For instance, the Nambu - Jona - Lasinio model [15] has been used to describe what drives the spontaneous symmetry breaking, much like the theories for superconductivity. However, in the context of QCD, our present understanding of the spontaneous chiral symmetry breaking comes from the Banks - Casher formula, which provides a link between the chiral condensate and the spectral density. [2] Virtual quarks and antiquarks of opposite chirality are attracted to each other due to the strong interactions and destabilize the trivial vacuum state. A condensate is formed, which gives rise to a nonvanishing expectation value of the bilinear fermionic operator. So, if chiral symmetry is spontaneously broken by a non zero value of the condensate, the density of the quark modes in infinite volume does not vanish at the origin. Spectral density and low - lying Dirac modes calculation is very important in order to understand the dynamics of spontaneous chiral symmetry breaking. There are several articles and studies on the spectral density of the Dirac operator, such as in references [10,

${ }^{\star}$ Speaker, e-mail: rudina.zeqirllari@fshn.edu.al 
9, 6]. In these papers the Dirac modes number is calculated by approximating the operator for low - lying modes applying the spectral projector method. In our work we propose a spectral method for the determination of the spectral density of Dirac operator near the origin using Gauss - Lanczos quadrature and we can show the dynamical spontaneous chiral symmetry breaking using chiral lattice fermions (Boriçi - Creutz fermions). We utilise the optimal properties of Krylov subspaces in approximating the distribution of the eigenvalues of the Dirac operator. These properties allow us to use the Gauss - Lanczos quadrature in order to estimate the number of modes of the Dirac operator and then the effective chiral condensate. Being chiral fermions, Boriçi - Creutz fermions are more suitable to work with than any other type of lattice fermions.

\subsection{Boriçi - Creutz fermions and chiral condensate}

The fermion doubling problem is a problem that is encountered when naively trying to put fermionic fields on a lattice. The famous no-go theorem of Nielsen and Ninomiya [17]most clearly reveals the fate of lattice fermions. It states that lattice fermion actions with chiral symmetry, locality and other common features must produce degrees of freedom in multiples of two in a continuum limit. In order to solve this problem, several strategies are in use, such as:

- Wilson fermions - explicitly violate chiral symmetry [21]

- Domain wall or overlap fermions - very expensive [16]

- Staggered fermions - four flavours [13]

- Minimally doubled fermions [12, 20, 4, 7]

Minimally doubled fermions have been proposed as a strictly local discretization of the QCD quark action, which preserve an exact chiral symmetry for a degenerate doublet of quarks, the minimum doubling allowed by the Nielsen - Ninomiya theorem. A type of this kind of fermions are Boriçi Creutz fermions. The Dirac operator for the Boriçi - Creutz fermions in the momentum space is as below:

$$
D_{B C}(p)=\sum_{\mu}\left[i \gamma_{\mu} \sin p_{\mu}+i\left(\Gamma-\gamma_{\mu}\right) \cos p_{\mu}\right]
$$

which has two poles, $p_{1}=(0,0,0,0)$ and $p_{2}=(\pi / 2, \pi / 2, \pi / 2, \pi / 2)$. corresponding to the two physical flavors. Therefore, there is always a preferred direction in the euclidean space time, given by the line that connects these poles (the major hypercube diagonal). So, this action cannot maintain a full hyper - cubic symmetry. [3] In order to restore the broken hyper-cubic symmetry, Capitani et al, [5] performed some perturbative calculations and proposed to add some counterterms to this action. In this work we have considered just one of them and the action now can be written as:

$$
D_{B C}(p)=\sum_{\mu}\left[i \gamma_{\mu} \sin p_{\mu}+i\left(\Gamma-\gamma_{\mu}\right) \cos p_{\mu}\right]+i\left(c_{3}-2\right) \Gamma
$$

\subsection{The effective condensate}

The Banks - Casher relation links the spectral density $\rho(\lambda, m)$ of the Dirac operator to the condensate as:

$$
\lim _{\lambda \rightarrow 0} \lim _{m \rightarrow 0} \lim _{V \rightarrow \infty} \rho(\lambda, m)=\frac{\Sigma}{\pi}
$$


where

$$
\Sigma=-\lim _{m \rightarrow 0} \lim _{V \rightarrow \infty}\langle\bar{\psi} \psi\rangle
$$

This relation can be read in both directions: a non zero spectral density implies that the symmetry is broken by a non vanishing $\Sigma$ and vice - versa. As we mentioned above, spectral density and low lying Dirac modes are very important for understanding spontaneous chiral symmetry breaking. Furthermore the chiral condensate is a useful order parameter to distinguish the phases at $T=0$, for minimally doubled fermions $[12,20,7,4]$. In the continuum theory and for small masses, the mode number can be calculated analytically in chiral perturbation theory. At present the chiral expansion of the spectral density is known to next-to leading order of chiral perturbation theory $[19,18,8]$. In infinite volume, chiral perturbation theory yields an expansion of the spectral density $\rho(\lambda, m)$ essentially in powers of $\lambda$ and $m$. The leading term is given by the Bank - Casher formula which relates the mode number $v$ to the "effective chiral condensate" defined through

$$
\Sigma_{\text {eff }}=\frac{\pi}{2} \frac{v(\Lambda)}{V}
$$

where $\Lambda=\sqrt{M^{2}-m^{2}}$. So, instead of using spectral density, the average number $v(\Lambda)$ of eigenmodes of the massive hermitian operator $D^{\dagger} D+m^{2}$ with eigenvalues $\alpha \leq M^{2}$, seems to be a more convenient quantity to deal with. Since:

$$
v(\Lambda)=V \int_{-\Lambda}^{\Lambda} d \lambda \rho(\lambda, m)
$$

the mode number carries the same information as the spectral density $[10,18,8]$. In the presence of a given gauge field, the number of eigenmodes of $D_{m}^{\dagger} D_{m}$ with eigenvalues $\alpha \leq M^{2}$, can be determined by calculating the eigenvalues and their multiplicities numerically.

\section{Methodology}

As we mention above, virtual quarks and antiquarks of opposite chirality are attracted to each other due to the strong interactions and destabilize the trivial vacuum state. A condensate is formed, which gives rise to a nonvanishing spectral density of Dirac operator modes. So, the presence of the chiral condensate is a clear fact of the SCHSB. When hypercubic symmetry is broken, like in the BC fermions case, the total momentum of the pair quark-antiquark of opposite chirality is not zero, because the velocities are different in different directions. So for the pair creation is required energy. In this way, the absence of hypercubic symmetry prohibits the creation of such pairs, so there is no condensate. Therefore the chiral symmetry is exact. What we can think is that the conditions for having a chiral condensate, can be fulfilled only if the Lorentz symmetry is present. Using this fact, by changing the counteterms $c_{3}$ in the $\mathrm{BC}$ action, we can find the proper values of $c_{3}$ which allows us to be in the proper phase, the phase with spontaneous chiral symmetry breaking. The values of $c_{3}$ that fulfill the conditions above, are the ones which restore the hypercubic symmetry. We have chose seven different values of $c_{3}=0.1,0.2,0.35,0.4,0.6,0.8,1$ and performed our calculations in the quenched approximation in a $24^{4}$ lattice.

\subsection{The algorithm for chiral condensate evaluation}

In this section, we show an alternative way to count efficienty the mode number and estimate the effective condensate. We utilise the optimal properties of Krylov subspaces in approximating the 
distribution of the eigenvalues of the Dirac operator. These properties allows us to use the Gauss Lanczos quadrature in order to estimate the number of modes of the Dirac operator. What we propose is to use Lanczos algorithm in order to produce the tridiagonal and symmetric Lanczos matrix $T_{n}$. Its eigenvalues, or the so called Ritz values, tend to approximate the eigenvalues of the original matrix $A_{n}=D_{n}^{\dagger} D_{n}$. The eigenvalues and eigenvectors of a symmetric and tridiagonal matrix can be computed by the QR method [11, 1]. In fact, Ritz values are the nodes of the Gauss - Lanczos quadrature with the corresponding weights being the first elements of the Ritz vectors squared. Bai, Fahey and Golub [1] observed that a vector-matrix-vector of the form $\psi^{\dagger} f(A) \psi$ can be expressed as an integral of the function $f$ (.) over a modified spectral measure. An n-point Gaussian quadrature approximation to this integral is then given by:

$$
\psi^{\dagger} f(A) \psi \approx \sum_{i=1}^{n} \omega_{i} f\left(\theta_{i}\right)
$$

where the abscissa, $\theta_{i}$, of this quadrature rule are given by the Ritz values. These are generated by the Lanczos algorithm applied with seed vector $\psi$, while the weights $\omega_{i}$ are the squares of the first components od the corresponding eigenvectors of $T_{n}$.

So, using Gauss - Lanczos quadrature we calculate the distribution of the eigenvalues modes, and then we find the low lying Dirac modes as:

$$
\frac{v(\Lambda)}{V}=\sum_{i=1}^{k(\Lambda)} \omega\left(\theta_{i}\right)
$$

since our function $f\left(\theta_{i}\right)$ is the Heaviside function:

$$
f\left(\theta_{i}\right)= \begin{cases}1 & i \leq k(\Lambda) \\ 0 & i>k(\Lambda)\end{cases}
$$

Let $A \epsilon^{N \times N}$ be a hermitian matrix and $b \in^{N}$ a starting vector. Then the following algorithm computes the Gauss - Lanczos quadrature $[14,1]$

Compute $\alpha_{i}$ and $\beta_{i}$ using Lanczos algorithm for $A x=b$

Set $\left(T_{n}\right)_{i, i}=\alpha_{i},\left(T_{n}\right)_{i+1, i}=\left(T_{n}\right)_{i, i+1}=\beta_{i}$ otherwise $\left(T_{n}\right)_{i, j}=0$

Compute eigenvalues $\lambda_{i}$ and eigenvectors $v_{i}$ of $T_{n}$, where $i=1 \ldots . n$

Sort eigenvalues and eigenvectors in the increasing order of eigenvalues

Set $k$ as the maximum index which correspond to the cut-off eigenvalue

Set $\theta_{i}$ to the positive square root of the original eigenvalues

Set $z_{i}$ the first element of eigenvectors $v_{i}$ where $i=1 \ldots . . n$

Set $\omega_{i}=z_{i}^{2}$

Compute the mode number $v_{k}=\sum_{i=1}^{k} \omega_{i}$

Algorithm 1: Algorithm for the Gauss - Lanczos quadrature

\section{Results}

In order to test our method we chose to work with minimally doubled fermions (BC fermions) in the quenched approximation, for the simple fact that this kind of fermions are chiral and we can easily demostrate the dynamical spontaneous chiral symmetry breaking. As we will see below the counterterm $c_{3}$ mentioned above, is a kind of order parameter, which divides two clear phases (the one with 


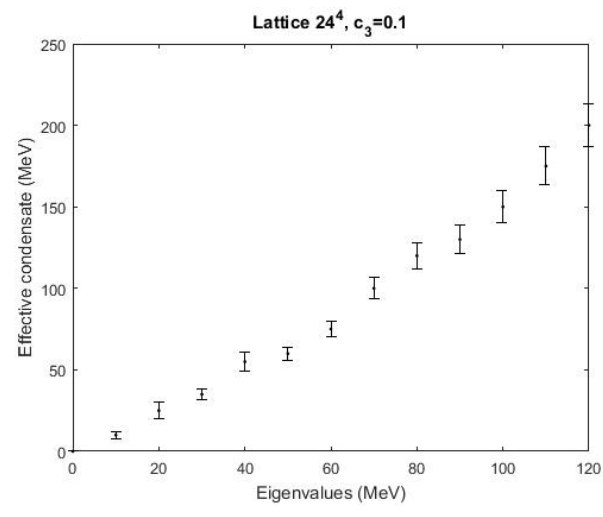

(a) $c_{3}=0.1$

The calculations show that there is no formation of chiral condensate

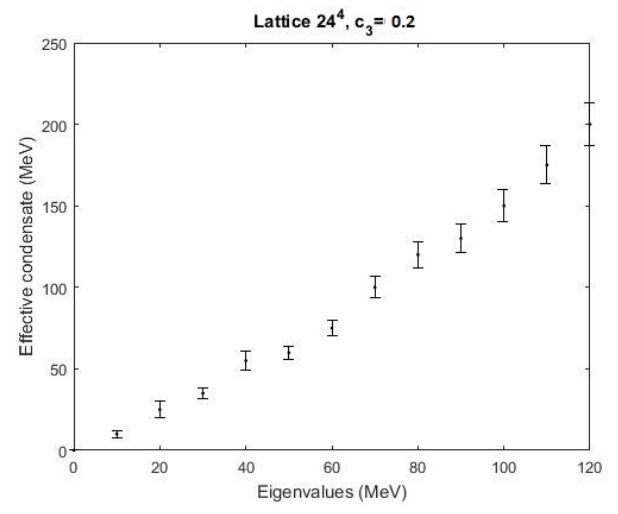

(b) $c_{3}=0.2$

The calculations show that there is no formation of chiral condensate

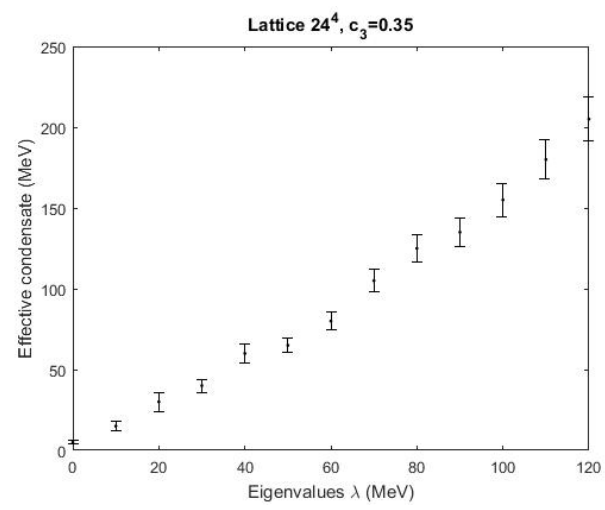

(c) $c_{3}=0.35$

The calculations show that there is a chance for the formation of chiral condensate

Figure 1. Effective condensate for different values of the counterterms $c_{3}<0.4$ added in $\mathrm{BC}$ action. It can be shown that for these values of the countertems, we don't have low-lying modes condensation

chiral symmetry and the other with SCHSB). Using Gauss - Lanczos quadrature we have calculated the modes number for minimally doubled fermions, and therefore the effective chiral condensate, in a background of 1000 gauge configurations, generated using the SU(3) theory. The results are averaged over 1000 configurations. As expected by the chiral perturbation theory, the low-modes of the operator condense and reach a "plateau". In order to find the effective condensate we have taken some "representative" data using the original data's histogram, and perform a linear fit of them, considering the fact that the datas have different weights. It's clear that for $c_{3}<0.4$ we have the phase with chiral symmetry, while for $c_{3} \geq 0.4$, are in the SCHSB phase. 


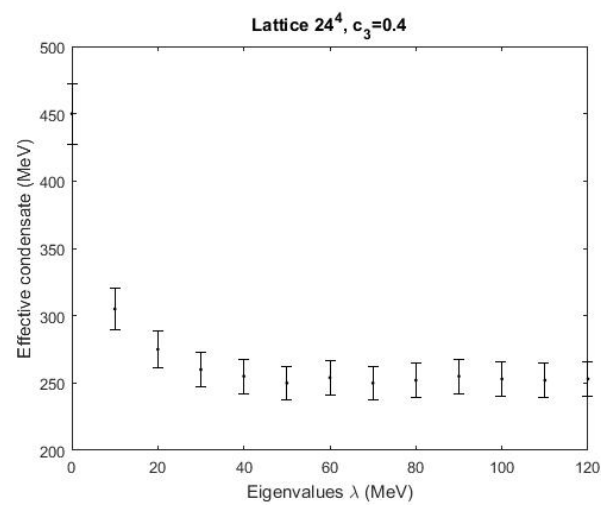

(a) $c_{3}=0.4$

Can be easily shown that for this value the low-lying modes condense, therefore there is a value of effective condensate different from zero

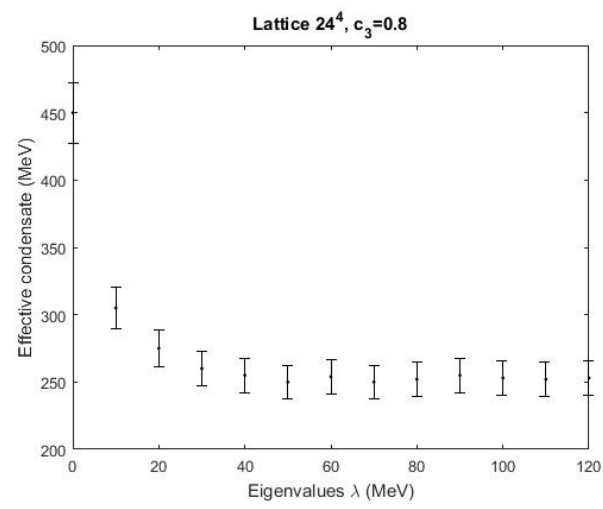

(c) $c_{3}=0.8$

It can be seen that there is a value of effective condensate different from zero

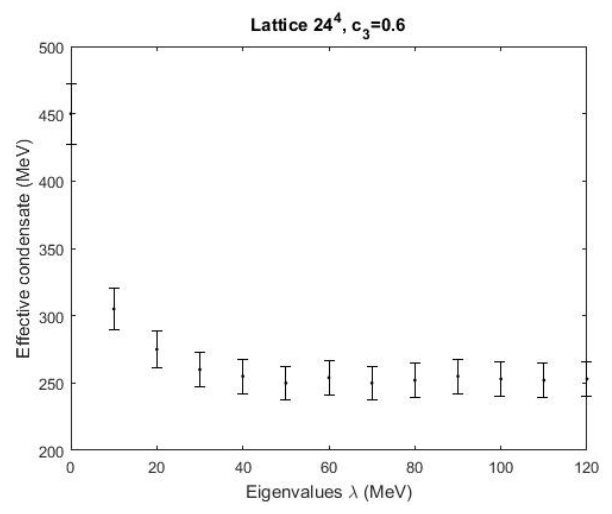

(b) $c_{3}=0.6$

It can be seen that there is a value of effective condensate different from zero

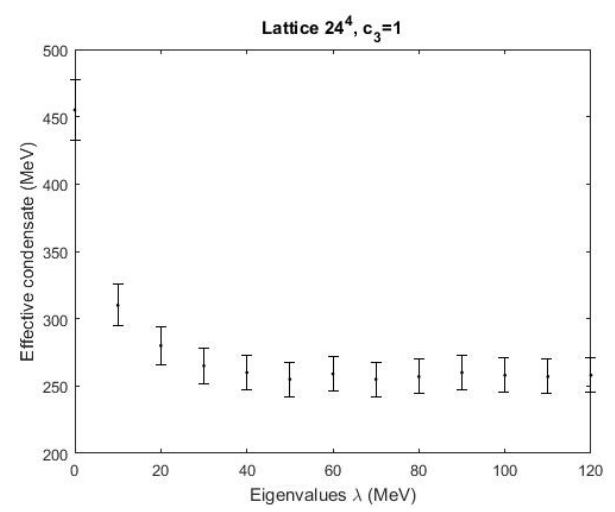

(d) $c_{3}=1$

It can be seen that there is a value of effective condensate different from zero

Figure 2. Formation of effective condensate for different values of the counterterms $c_{3} \geq 0.4$ added in $\mathrm{BC}$ action.

\section{Conclusions}

Chiral symmetry and spontaneous chiral symmetry breaking are very imporant in QCD. Using minimally doubled fermions (as chiral fermions) and Lanczos quadrature we can explore and understand the dynamical mechanism of spontaneous chiral symmetry breaking in a very simple way. The metodology we propose has a minimal numerical cost, because we utilise the optimal properties of Krylov subspaces in approximating the distribution of the eigenvalues of the Dirac operator, not in calculating every eigenvalue of the operator. One important thing of this work is the fact that the chiral condensate can be used as an order parameter for Boriçi - Creutz and help us to find the proper counterterms that restore partially the broken hypercubic symmetry. This work aims to the use of this methodology for further detailed studies of minimally doubled fermions. 


\section{Acknowledgement}

The simulations and calculations of this work are performed in the mini - server of the Faculty of the Natural Sciences, Department of Physics, provided by the project "Simulations of lattice chiral fermions" and in the paralel system of the Faculty of Information Technology, Polytechnic University of Tirana. Acknowlegdements to the maintenance staff and all the persons who made possible the access to the servers. 


\section{References}

[1] Zhaojun Bai, Gark Fahey, and Gene Golub. "Some large-scale matrix computation problems". In: Journal of Computational and Applied Mathematics 74.1-2 (1996), pp. 71-89.

[2] Tom Banks and A Casher. "Chiral symmetry breaking in confining theories". In: Nuclear Physics B 169.1 (1980), pp. 103-125.

[3] Paulo F Bedaque et al. "Broken symmetries from minimally doubled fermions". In: Physics Letters B 662.5 (2008), pp. 449-455.

[4] Artan Boriçi. "PoS (LATTICE 2008) 231 Minimally Doubled Fermion Revival”. In: (2008).

[5] Stefano Capitani et al. "Renormalization of minimally doubled fermions". In: Journal of High Energy Physics 2010.9 (2010), p. 27.

[6] Krzysztof Cichy, Elena Garcia-Ramos, and Karl Jansen. "Chiral condensate from the twisted mass Dirac operator spectrum”. In: Journal of high energy physics 2013.10 (2013), p. 175.

[7] Michael Creutz. "Four-dimensional graphene and chiral fermions". In: Journal of High Energy Physics 2008.04 (2008), p. 017.

[8] PH Damgaard et al. "The microscopic spectral density of the QCD Dirac operator". In: Nuclear Physics B 547.1-2 (1999), pp. 305-328.

[9] H Fukaya et al. "Determination of the chiral condensate from QCD Dirac spectrum on the lattice”. In: Physical review D 83.7 (2011), p. 074501.

[10] Leonardo Giusti and Martin Lüscher. "Chiral symmetry breaking and the Banks-Casher relation in lattice QCD with Wilson quarks". In: Journal of High Energy Physics 2009.03 (2009), p. 013.

[11] Gene H Golub and Charles F Van Loan. Matrix computations. Vol. 3. JHU Press, 2012.

[12] Luuk H Karsten. "Lattice fermions in euclidean space-time". In: Physics Letters B 104.4 (1981), pp. 315-319.

[13] John Kogut and Leonard Susskind. "Hamiltonian formulation of Wilson's lattice gauge theories”. In: Physical Review D 11.2 (1975), p. 395.

[14] Cornelius Lanczos. "Solution of systems of linear equations by minimized iterations". In: $J$. Res. Nat. Bur. Standards 49.1 (1952), pp. 33-53.

[15] Yoichiro Nambu and Giovanni Jona-Lasinio. "Dynamical model of elementary particles based on an analogy with superconductivity. I”. In: Physical Review 122.1 (1961), p. 345.

[16] Herbert Neuberger. "Overlap lattice Dirac operator and dynamical fermions". In: Physical Review D 60.6 (1999), p. 065006.

[17] Holger Bech Nielsen and Masao Ninomiya. "Absence of neutrinos on a lattice:(I). Proof by homotopy theory". In: Nuclear Physics B 185.1 (1981), pp. 20-40.

[18] JC Osborn, D Toublan, and JJM Verbaarschot. "From chiral random matrix theory to chiral perturbation theory". In: Nuclear Physics B 540.1-2 (1999), pp. 317-344.

[19] Andrei V Smilga and J Stern. "On the spectral density of Euclidean Dirac operator in QCD". In: Physics Letters B 318.3 (1993), pp. 531-536.

[20] Frank Wilczek. "Lattice fermions”. In: Physical review letters 59.21 (1987), p. 2397.

[21] Kenneth G Wilson. “Confinement of quarks”. In: Physical Review D 10.8 (1974), p. 2445. 